\title{
Fiber-Optic Temperature Sensor Based on Single Mode Fused Fiber Coupler
}

\author{
Kwang Taek Kim* and Kiu Ha Park \\ Department of Optoelectronics, Honam University 59-1, Seobong-Dong, Gwangsan-Gu, \\ Gwangju 505-714, Korea
}

(Received August 11, 2008 : accepted August 26, 2008)

\begin{abstract}
This paper reports a fiber-optic temperature sensor using a single mode fused fiber coupler incorporating a thermo-optic external medium. The spectral transmission was altered by changing the refractive index of the external thermo-optic medium. A theoretical and experimental investigation was carried out with the aim of achieving high sensitivity. The measured sensitivity for the environmental temperature was as high as $-1.5 \mathrm{~nm} /{ }^{\circ} \mathrm{C}$.
\end{abstract}

Keywords : fused fiber coupler, fiber-optic temperature sensor, thermo-optic effect, spectral transmission

OCIS codes : (060.1810) Couplers, switches, and multiplexers, (060.2340) Fiber optics components, (060.2370) Fiber optics sensors

\section{INTRODUCTION}

Fused fiber couplers are key devices in optical communications and optical fiber sensor systems that either combine or split optical signals [1-5]. The continuous in-line structure of these devices offers many attractive features, such as a low insertion loss, low back reflection, good mechanical reliability, and no interface problems with other fiber systems. A typical $2 \times 2$ fused fiber coupler is made by heating and pulling two single mode fibers. The two separate fibers are fused into a combined and tapered body. The fused fiber coupler can perform many important functions, including optical power dividing, wavelength selective coupling and polarization splitting $[6,7]$. The desired characteristics of the coupler can be controlled through proper control of the tapered length. The spectral selectivity is an important property to achieve high sensitivity, high precision and high reliable sensing technology because the spectral response is immune to any optical source instability or optical loss in the fiber [8].

This study examined the feasibility of a $2 \times 2$ fused fiber coupler as a fiber optic sensor based on its spectral transmission. The taped region was covered with a thermo -optic external medium. The key hypothesis was that the

*Corresponding author: ktkim@honam.ac.kr transmission and coupling characteristics of the coupler can interact with the external medium through evanescent wave coupling. The environmental temperature affects the refractive index of the external medium, which can cause a shift in spectral transmission. Theoretical analysis predicted the device structural condition needed to achieve high sensitivity and explained the behavior of the sensor device. The feasibility for practical applications is also discussed.

\section{THEORETICAL CONSIDERATIONS}

Fig. 1 shows a schematic diagram of the proposed temperature sensor using a $2 \times 2$ fused single fiber coupler incorporating a thermo-optic external medium. Two fibers were combined and tapered adiabatically by heating and pulling. Evanescent wave coupling between two fibers occurs in the waist region. The tapered region was surrounded by a new external medium whose refractive index was sensitive to the environmental temperature. For simple analysis, the device was modeled into a planar waveguide directional coupler. In the calculation, it was assumed that the core width was reduced adiabatically from $8.0 \mu \mathrm{m}$ to $1.0 \mu \mathrm{m}$ during tapering. The symbols, $n_{c o}, n_{c l}$ and $n_{e x}$ denote the refractive index of the core, cladding and external medium, respectively. The 
refractive index of the core and cladding were assumed to be 1.449 and 1.444 , respectively. The chromatic dispersion of the materials was not considered.

In the directional coupler, optical coupling between the adjacent two waveguides was repeated periodically during propagation in the coupling region and the optical power was divided into two output ports, the throughput and cross coupled ports. The optical coupling ratio is dependent on many parameters including the geometry of the taper region, the refractive index of the external medium and the optical wavelength. Figs. 2 shows the phenomenon of mode coupling(left) and power dividing(right) between the two waveguides for different wavelengths and for different refractive indexes of the external medium. A comparison of Fig. 2(a) with 2(b) showed that evanescent wave coupling between two adjacent waveguides was repeated more rapidly along the fiber direction when the wavelength is longer. This was attributed to the mode field distribution increasing with

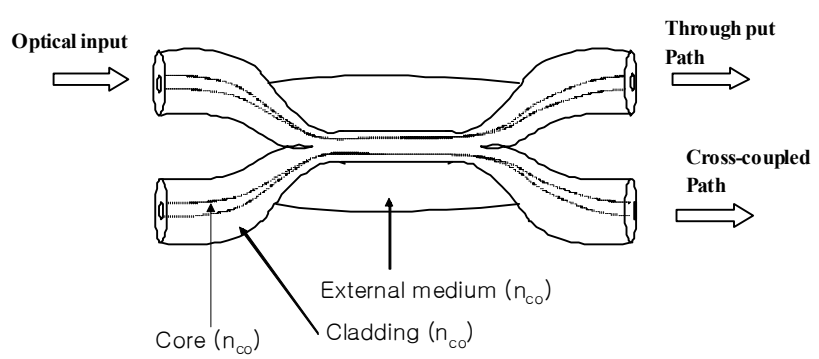

FIG. 1. Structure of fiber-optic sensor based on fused fiber coupler incorporating thermo-optic external medium. longer wavelength. Therefore, the fused fiber coupler has wavelength selective properties. Fig 2(b) and 2(c) show the effect of the refractive index of the external medium on the coupling characteristics. The $n_{e x}$ considered in Figs. 2(b) and (c) was 1.444 and 1.440, respectively. A lower $n_{e x}$ results in stronger coupling.

Fig. 3 shows the calculated spectral transmission of the throughput port at various $n_{e x}$. The results reveal that the peak maximum and minimum wavelengths in the spectral transmission shift to a shorter region, and the interval between the two peak points increased with increasing refractive index of the external me$\operatorname{dium}\left(n_{e x}\right)$. In addition, it was found that the peak wavelength shift $(\Delta \lambda)$ per unit change in refractive index of the external medium $\left(\Delta n_{e x}\right)$, i.e. $\left(\Delta \lambda / \Delta n_{e x}\right)$, was not uniform. For example, the transmission spectrum for two cases, $n_{e x}=1.424$ and 1.429 , were similar, while in another two cases, $n_{e x}=1.442$ and 1.444 , the two spectra were quite different, even though the difference was small, $\Delta n_{e x}$. The highest $\Delta \lambda / \Delta n_{e x}$ was observed when $n_{e x}$ was close to $n_{d}$, which is the refractive index of the fiber cladding. It can be predicted that a change in refractive index of the thermo-optic external medium causes a shift in the output transmission spectrum. In order to acquire high sensitivity, an external medium with a high thermo-optic coefficient and suitable refractive index should be selected.

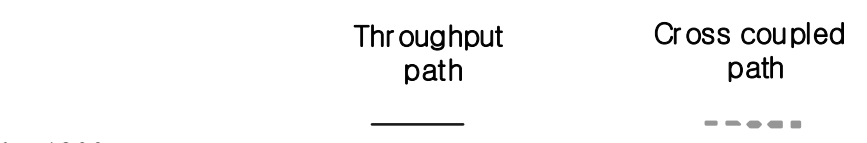

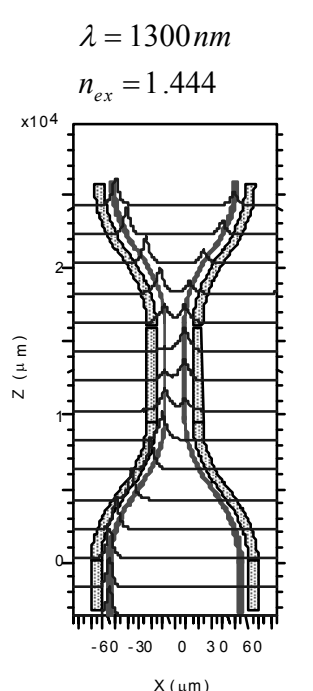

(a)

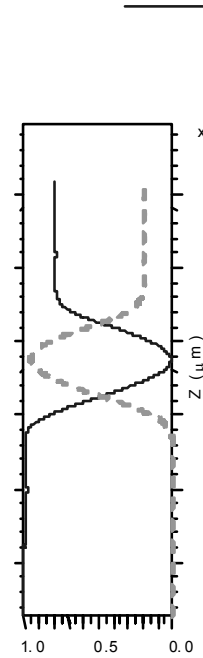$$
\text { (1.0.0. }
$$

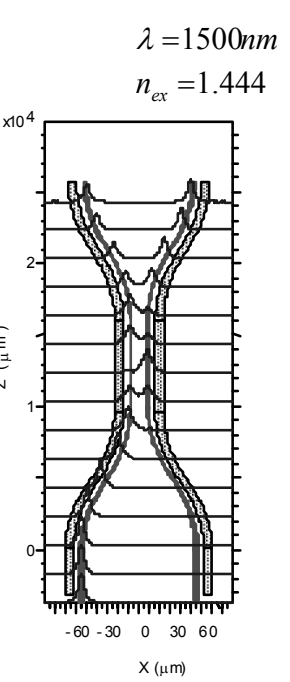

(b)
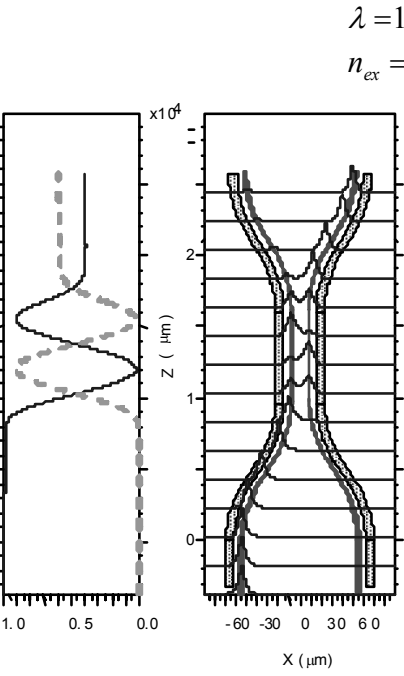

$\mathrm{X}(\mu \mathrm{m})$

(c)

FIG. 2. Phenomenon of mode coupling(left) and power dividing(right) for different wavelength( $\lambda$ ) and $n_{\text {ex }}$., (a) $\lambda=1300 \mathrm{~nm}$, $n_{e x}=1.444$, (b) $\lambda=1500 \mathrm{~nm}, n_{e x}=1.444$, (c) $\lambda=1500 \mathrm{~nm}, n_{e x}=1.440$ 


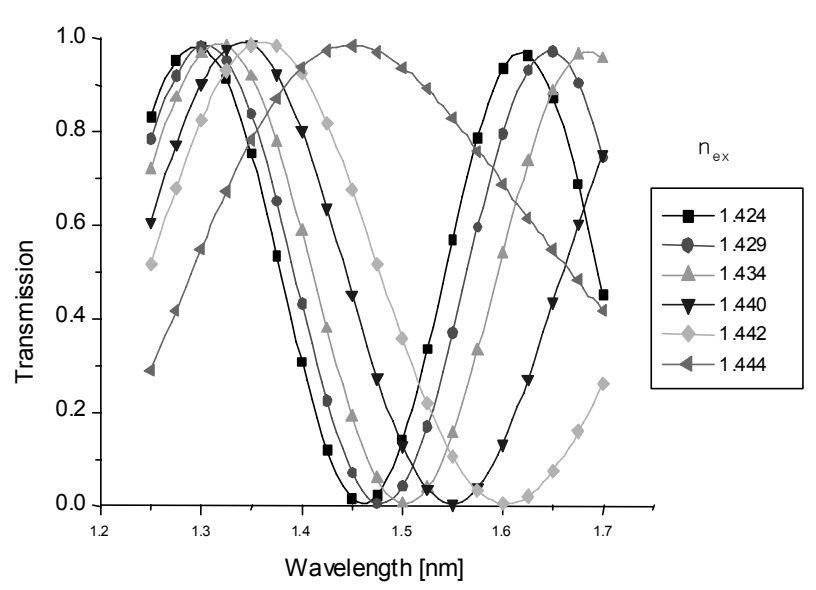

FIG. 3. Calculated transmission spectrum for various $n_{e x}$

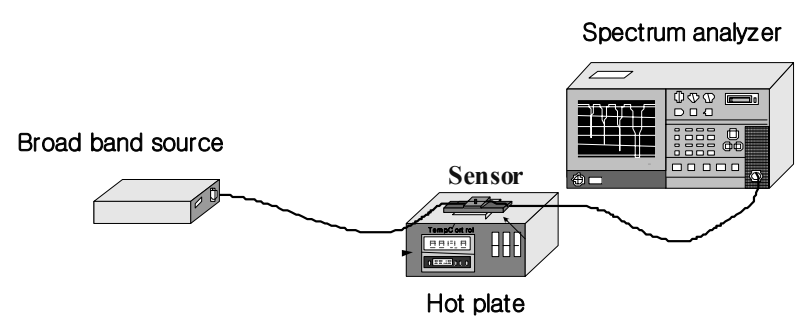

FIG. 4. Experimental set up for measuring the transmission spectrum with temperature variation.

\section{EXPERIMENTAL RESULTS}

A standard single mode fiber (SMF 28) was used in the experiment. Two fibers were aligned, heated with a flame from two micro torches and tapered by pulling. The maximum peak wavelengths of the throughput and crossed coupled ports were adjusted to $1550 \mathrm{~nm}$ and $1310 \mathrm{~nm}$, respectively, without an external medium. After this process, the sample was mounted on a U shaped quartz tube, $40 \mathrm{~mm}$ in length, using UV curing epoxy. Since one side of the quartz tube is opened, an external medium can be inserted into the blank area of the quartz tube. As a result, the tapered region of the coupler is surrounded by the external medium. Before measuring the performance of the sensor device, three types of liquids were prepared for the external medium. The refractive indices of these materials were measured using a prism coupler at a wavelength of $1550 \mathrm{~nm}$. The prepared materials were water $\left(n_{e x}=1.32\right)$, and index matching liquids made from water and glycerin $\left(n_{e x}=\right.$ 1.397 and 1.440). The experimental set-up consisted of a broadband source, hotplate, and spectrum analyzer, as shown in Fig. 4. Initially, the spectral transmission was measured for each prepared external medium (Fig. 5). Only one device was used in this experiment. Therefore, the other device conditions were constant.

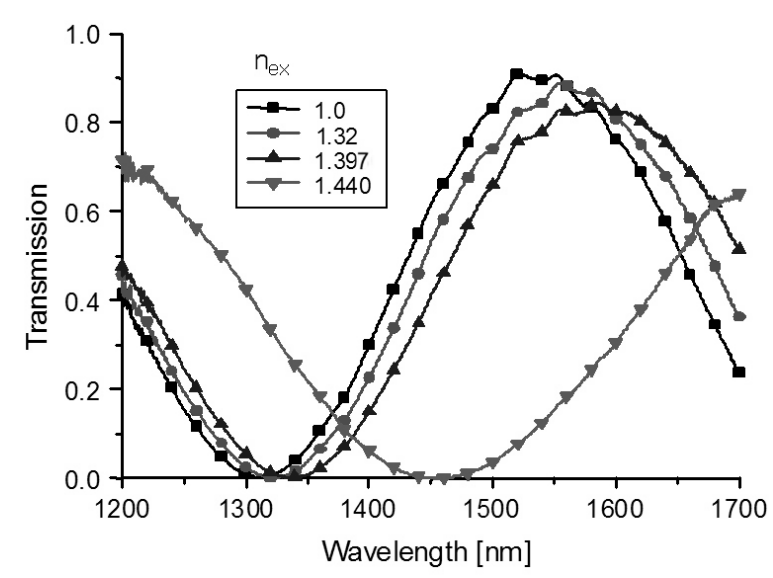

FIG. 5. Measured spectrum of the device for different $n_{e x}$

As theoretically expected, the experimental results showed a shift in the peak positions of the transmission spectrum to a longer wavelength region with increasing $n_{e x}$. See the spectra corresponding to the air and water media. Despite the large difference in index between them, the difference in peak wavelength was small. However, for two cases, $n_{e x}=1.397$ and 1.440 , the two spectra were quite different, even though there was a small difference in $n_{e x}$. For the cases of air, water and a matching liquid with smaller index, the evanescent wave cannot extend deeply into the external medium because the refractive index of the external medium was much lower than that of the fiber cladding(1.444). As the refractive index of the external medium approaches that of the fiber cladding, the evanescent wave penetrates more deeply into the external medium and the coupling characteristic of the two fibers become sensitive to small changes in the refractive index of the external medium.

In order to measure the temperature sensitivity, the devices were packaged into a steel tube after inserting them into an external medium. Both ends of the steel tube were sealed by epoxy to prevent leakage of the liquids. In the first device, no external medium was inserted. In the second, third, and fourth devices, water and two index mismatching liquids $\left(n_{e x}=1.397\right.$ and 1.440) were used as the external medium. In order to evaluate the coupler as a fiber-optic temperature sensor, the environmental temperature was controlled using a hot plate. Fig. 6(a), (b), (c), and (d) show the transmission spectrum with temperature of the four devices. When the taped region was surrounded by air, there was virtually no change in the wavelength responses with temperature, as shown in Fig. 6(a). This means that the fused fiber coupler is stable against environmental temperatures without an external medium. The $n_{c o}$ and $n_{c l}$ are expected to increase with increasing temperature due to the positive thermo-optic(T-O) coefficients of the materials, including $\mathrm{GeO}_{2}$-doped silica and pure silica [8]. However, 


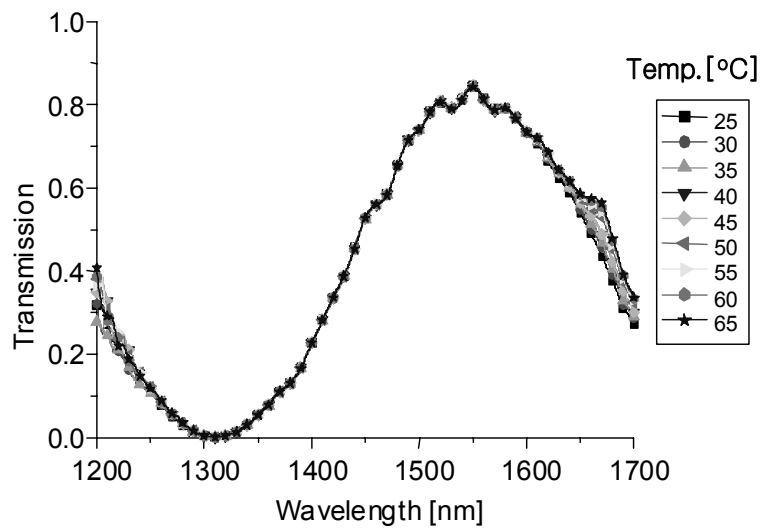

(a)

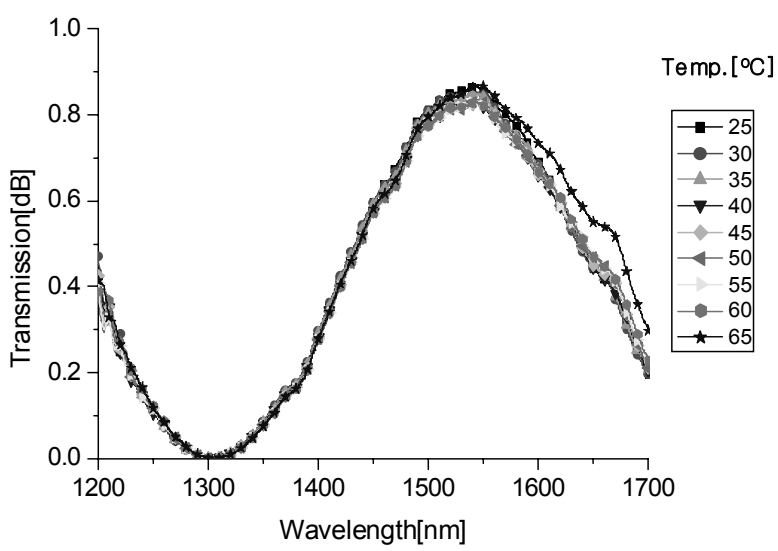

(c)

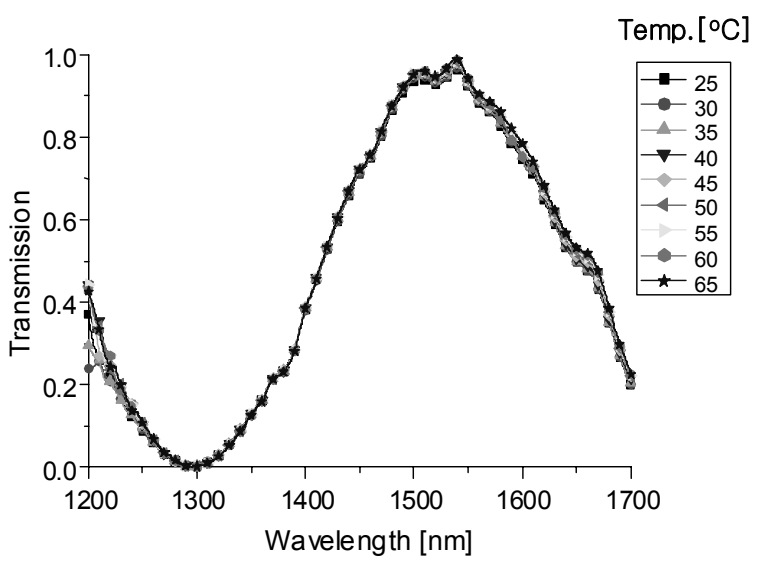

(b)

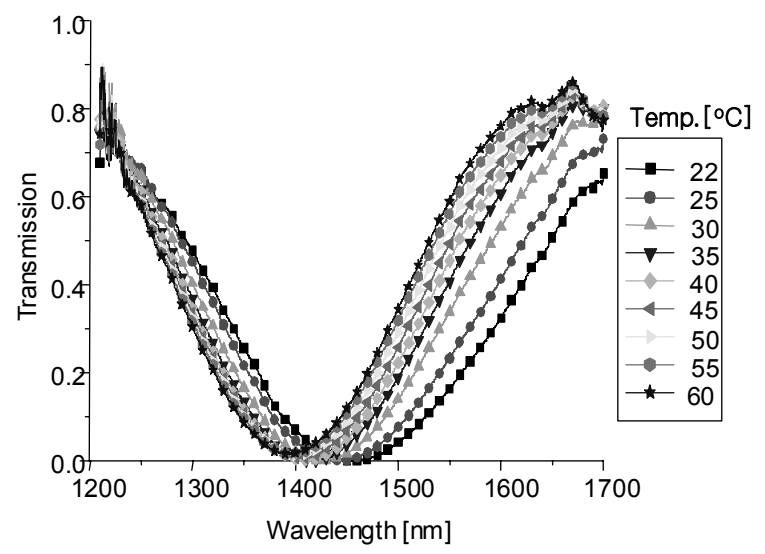

(d)

FIG. 6. Measured transmission spectrum in accordance with environmental temperature, (a) $n_{e x}=1.0$ (air), (b) $n_{e x}=$ 1.32 (water), (c) $n_{e x}=1.397$ (water and glycerin mixture), (c) $n_{e x}=1.440$ (water and glycerin mixture).

the T-O coefficients of those materials are too small to affect the transmission spectrum by temperature variations.

For the other two materials, water and the index mismatching liquid $\left(n_{e x}=1.397\right)$, the device also did not show any increase in temperature sensitivity. In this case, although the refractive index of the external medium varies with temperature, the refractive index variation in the low index region does not influence the coupling characteristics, as predicted theoretically.

From Fig. 6(d), the highest temperature sensitivity was observed when an index matching liquid with the refractive index closest to that of the fiber cladding was used. The thermo-optic coefficient was approximately $-3 \times 10^{4}$. The peak wavelength shift per unit temperature variation was $-1.5 \mathrm{~nm} /{ }^{\circ} \mathrm{C}$. The sensitivity of the device depends on the T-O coefficient of the external medium as well as the refractive index of the medium. Various materials can be candidates as external media for many types of materials, such as polymers.

\section{DISCUSSION}

There are several in-line evanescent wave technologies such as tapering [9], cladding etching [10] and sidepolishing [11]. The transmission of the devices is commonly affected by the refractive index of the outside medium. In order to impose spectral selectivity to those device, additional complicated work is needed, such as engraving a Bragg grating for etched fibers, and planar waveguide coating for side-polished fibers. The single mode taper fiber has many advantages for fiber optic sensors, including simple fabrication, high reproducibility and high reliability. However, it has no spectral selectivity for sense applications.

In this study, a single mode fused fiber coupler was used as an evanescent wave sensor with spectral selectivity. The device behaved like a directional coupler. The fabrication produces of the coupler is simple and similar with that of a single mode tapered fiber. The coupler has two output ports. Either(both) the throughput port or(and) the cross-coupled port can be used to detect the output spectrum. The information regarding 
temperature can be obtained from either the spectral width or peak wavelength of the detected spectrum. Furthermore, the device can operate as an intensity modulation sensor when a single wavelength optical source is used. The key features of the sensor include high sensitivity, simple fabrication and high mechanical stability as well as the capability of remote sensing. The operating temperature range depends entirely on the thermal durability of the external medium.

\section{CONCLUSION}

This paper reports an in-line fiber optic temperature sensor based on a single mode fused fiber coupler. It was predicted theoretically that the transmission spectrum of the coupler would be sensitive to the environmental temperature when the fused tapered region is surrounded by an external medium. The behavior of the device was explained by modeling the device as a planar waveguide coupler. The device structural condition was found to have high sensitivity through theoretical analysis and experiment. The experimental results were in good agreement with theoretical analysis. The fabricated device showed a sensitivity of $-1.5 \mathrm{~nm} /{ }^{\circ} \mathrm{C}$. It is anticipated that the sensor will find applications as a temperature sensor on account of the easy fabrication process, high reproduction property and mechanical reliability.

\section{REFERENCES}

[1] B. P. Pal "Fabrication and Modeling of Fused Biconical Tapered Fiber Couplers", Fiber and integrated optics, vol. 22, no. 2, pp. 97-117, 2003.
[2] B. E. Olsson and P. A. Andrekson, “A simple method for loss and coupling ratio determination in fused fiber couplers," IEEE Photon. Technol. Lett, vol. 8, no. 3, pp. 399-401, 1996.

[3] T. Tazawa, T. Kanie, and M. Katayama, "Fiber-optic coupler based refractive index sensor and its application to biosensing," Appl. Phys. Lett. vol. 91, 113901-113903, 2007.

[4] C. Bariain. I. R, F. J. Arregui, and M. Lopez-Amo, "Optical fiber humidity sensor based on a tapered fiber coated with agarose gel," Sensors and Actuators. B, Chemical vol. 69, no. 1/2, pp. 127-131, 2000.

[5] R Chen, G F Fernando, T Butler and R. A. Badcock "A novel ultrasound fibre optic sensor based on a fused -tapered optical fibre coupler," Measurement Science 8 Technology, vol. 15, no. 8, pp. 1490-1495, 2004.

[6] T. Bricheno and V. Baker "All-fiber polarization splitter/ combiner,” Electronic Letters, vol. 21, no. 6, pp. 251-252, 1985.

[7] M. S. Yataki, D. N. Payne, and M. P. Varnham, "Allfibre polarizing beamsplitter," Electronics Letters, vol. 26. no. 6, 249-251, 1985.

[8] A. Wang, K. A. Murphy "Optical-fiber temperature sensor based on differential spectral reflectivity," Smart Materials \& Structures, vol. 1, no. 1, pp. 5-7, 1992.

[9] Izawa, T., and Sudo, A., Optical fibers: materials and fabrication (KTK Scientific Publishers, Tokyo, 1987.

[10] J. Villatoro, D. Monzon-Hernandez, and E. Mejia "Fabrication and modeling of uniform-waist single-mode tapered optical fiber sensors," Appl. Opt., vol. 42, no. 13, pp. 2278-2283, 2003.

[11] B. Yun, N. Chen, and Y. Cui, "Highly Sensitive LiquidLevel Sensor Based on Etched Fiber Bragg Grating," IEEE Photon. Technol. Lett. : P, vol. 19, no. 21, pp. 1747-1749, 2007.

[12] K. T. Kim, K. H. Lee, and S. Hwangbo, "A refractometer based on fiber-to-liquid planar waveguide coupler," Sensors and Actuators A, Physical, vol. 126, no. 2, pp. 335339, 2006. 